\title{
Development of an Efficient Route to 2-Ethynylglycerol for the Synthe- sis of Islatravir
}

\author{
Stephan M. Rummelt ${ }^{\star}, J_{i} \mathrm{Qi}^{*} \star$, Yonggang Chen*, James F. Dropinski ${ }^{\star}$, Gregory Hughes ${ }^{\star}$, Jeffrey T. \\ Kuethe ${ }^{\ddagger}$, Donghong Li ${ }^{\#}$, Kevin M. Maloney \\ Christopher C. Nawrat ${ }^{\ddagger}$, Justin A. Newman ${ }^{\ddagger}$, Honggui Ouyang ${ }^{\S}$, Niki R. Patel ${ }^{\ddagger}, Z^{*}$ hen Qiao $^{\#}$, Gao \\ Shang ${ }^{\ddagger}$, Eric Sirota $\$$, Zhiguo Jake Song ${ }^{\ddagger}$, Lushi Tan $\$$, Richard J. Varsolona ${ }^{\ddagger}$, Baoqiang Wan ${ }^{\S}$, Brian M. \\ Wyvratt ${ }^{\ddagger}$, Feng Xu+, Yingju Xu*, Jingjun Yin ${ }^{\ddagger}$, Shaoguang Zhang ${ }^{\ddagger}$, Ralph Zhao \\ ¥ Department of Process Research and Development, Merck \& Co., Inc., Rahway, New Jersey 07065, United States \\ $\S$ Process R\&D, WuXi AppTec Co., Ltd., 288 Fute Zhong Road, Shanghai 200131, China \\ \# Department of Synthetic Chemistry, Pharmaron Beijing Co., Ltd. 6 Taihe Road BDA, Beijing, 100176, China
}

\begin{abstract}
The unnatural, alkyne-containing nucleoside analog islatravir (MK-8591) is synthetically accessed through a biocatalytic cascade starting from 2-ethynylglycerol as a building block. Herein, we describe the development of an efficient synthesis of this building block including the initial route, route scouting and final process development. Key challenges that have been overcome are the development of an efficient and safe acetylenic nucleophile addition to an appropriate ketone, and the identification of a 2ethynylpropane-1,2,3-triol derivative with favorable physical properties. An acid-catalyzed cracking of commercially available 1,3dihydroxyacetone dimer and subsequent 1,2-addition of an acetylenic nucleophile has been discovered and optimized into the manufacturing process.
\end{abstract}

\section{INTRODUCTION}

The alkyne moiety is one of the two unnatural structural features in the nucleoside analog islatravir (1, MK-8591) and enters the biocatalytic cascade of the manufacturing route in the form of 2-ethynylglycerol 2 (Scheme 1). ${ }^{1}$ Retrosynthetic analysis of this key building block mostly relied on 1,2-addition of a metalated alkyne into a 1,3-disubstituted ketone. While appearing straightforward at first sight, the most direct approach of ethynyl addition into 1,3-dihydroxyacetone (DHA) is complicated by the two protic primary hydroxy groups and the dimeric nature of the starting material. Furthermore, the ethynyl source needed to be chosen carefully, as acetylene is a highly explosive gas and its use or generation are highly undesirable in a pharmaceutical manufacturing process. ${ }^{2}$ The challenging physical properties of triol $\mathbf{2}$ combined with the purity requirements demanded by the downstream biocatalytic cascade towards inorganic and organic impurities, constituted an additional key driver for route selection and development. ${ }^{1}$ Here, we describe our efforts to develop a short and efficient synthesis of triol 2 leading from a inital supply route, through route scouting, and to the manufacturing process.

Scheme 1. Biocatalytic Cascade for the Synthesis of Islatravir

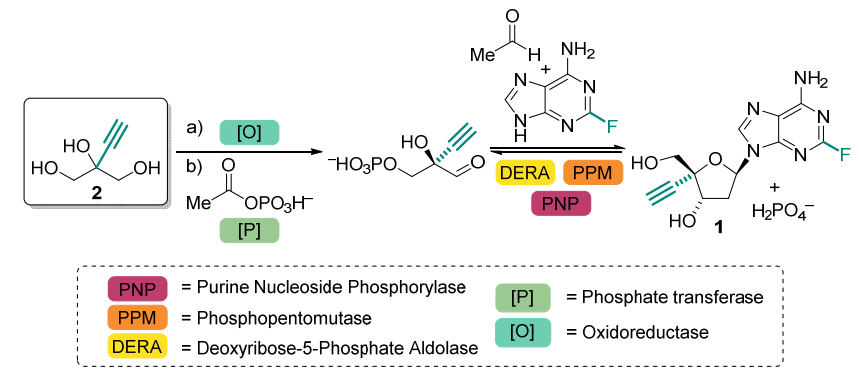

\section{RESULTS AND DICUSSION}

Properties and Crystalline Forms of Triol 2. At the outset of the project it was crucial to obtain information about the physical properties of triol 2 . The biocatalytic cascade for the commercial synthesis of islatravir (1) starts with triol $\mathbf{2}$ as the key building block (Scheme 1). Triol 2 therefore marks the transition from traditional synthetic chemistry to a biocatalytic reaction setup and thus occupies a strategically crucial position in the overall route. Hence, triol 2 or a suitable precursor should ideally be isolated in a form that exhibits physical properties suitable for storing and shipping, allowing flexibility in the supply chain. A crystalline intermediate would also provide a control point, which can ensure purity control before entering the biocatalytic cascade. Solid triol 2, obtained through crystallization at $-20{ }^{\circ} \mathrm{C}$, has a low melting point of $43{ }^{\circ} \mathrm{C}$ and deliquesces in air at ambient temperature (Figure 1). These unfavorable physical properties preclude the use of free triol $\mathbf{2}$ as an isolated intermediate. As triol $\mathbf{2}$ enters the biocatalytic cascade as a solution in water, intermediates that can be transformed to an aqueous solution of triol $\mathbf{2}$ in an operationally simple way would constitute a viable alternative. Due to the high solubility of triol 2 in water, efficient extraction from an organic phase into an 
aqueous phase is feasible. At the same time, extraction of triol $\mathbf{2}$ from an aqueous into an organic phase was found inefficient, prohibiting any process that relies on a standard aqueous workup to remove water soluble byproducts in the final step.

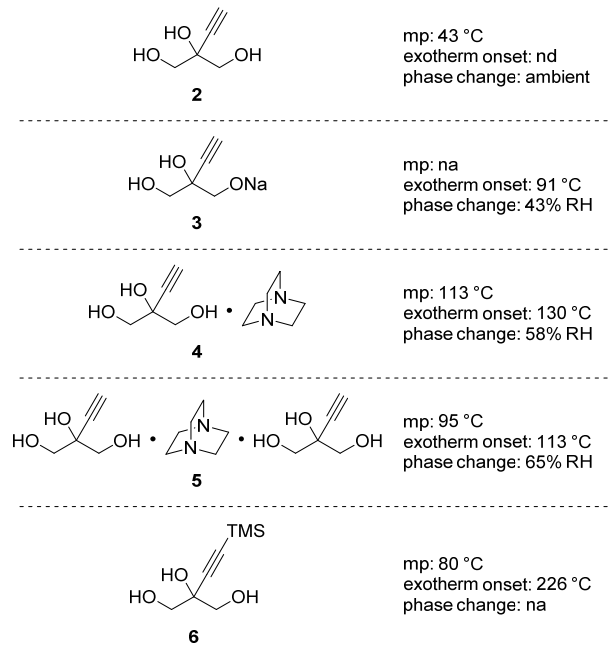

Figure 1. Solid, crystalline forms and derivatives of triol 2 and their physical properties. $\mathrm{mp}=$ melting point; $\mathrm{nd}=$ not determined; na $=$ not available; $\mathrm{RH}=$ relative humidity .

At the onset of our studies we aimed to identify alternative crystalline precursors of triol 2 (Figure 1). One strategy involved deprotonation of triol $\mathbf{2}$ and isolation of the corresponding alkoxide species. Addition of sodium methoxide to a solution of triol 2 in isopropyl alcohol results in the crystallization of a triol sodium salt $\mathbf{3}$, which can be isolated with $91 \%$ yield. While no melting point of the so obtained crystalline material could be determined, differential scanning calorimetry (DSC) analysis revealed a sharp exotherm of $369 \mathrm{~J} / \mathrm{g}$ with an onset temperature of $91{ }^{\circ} \mathrm{C}$. At a relative humidity (RH) of $43 \%$ the material underwent a phase change as observed by powder $\mathrm{x}$-ray diffraction (pXRD) and it ultimately turned deliquescent at 55\% relative humidity. While the physical properties of the triol sodium salt 3 at ambient conditions were improved compared to free triol 2, the strong exothermic behavior with a low onset temperature combined with the hygroscopicity are not ideal for storage, shipping and handling. Crystalline materials with similar unfavorable physical properties were also obtained when lithium or potassium salts were generated by a similar crystallization process. (see SI for further details).

Alternatively, triol 2 could be isolated as DABCO co-crystal $4(\mathrm{DABCO}=1,4$-diazabicyclo[2.2.2] $\mathrm{octane})$ after addition of DABCO (1.2 equiv) to a solution of triol 2 in THF in 93\% yield. DABCO complex 4 has a melting point of $113{ }^{\circ} \mathrm{C}$ and an exotherm of $1208 \mathrm{~J} / \mathrm{g}$ with an onset temperature of $130{ }^{\circ} \mathrm{C}$ as determined by DSC analysis. The pXRD spectrum showed no change up to a relative humidity of $58 \%$. In a slurry in 1-propanol at room temperature DABCO co-crystal 4 converts to hemiDABCO co-crystal 5. The hemi-DABCO co-crystal 5 melts at $95^{\circ} \mathrm{C}$ as determined by DSC and therefore has a melting point $52{ }^{\circ} \mathrm{C}$ higher than that of triol 2 itself and $18{ }^{\circ} \mathrm{C}$ lower than that of the mono-DABCO co-crystal 4. DSC again revealed an exotherm of $1482 \mathrm{~J} / \mathrm{g}$ with an onset temperature of $119^{\circ} \mathrm{C}$. Both DABCO co-crystals $\mathbf{4}$ and $\mathbf{5}$ were characterized by single crystal x-ray diffraction (see SI for details). An aqueous solution of free triol 2 with sufficient purity for the downstream biocatalytic cascade could be accessed through treatment of an aqueous solution of the triol sodium salt $\mathbf{3}$ or DABCO co-crystals $\mathbf{4}$ and
5 with an acidic resin. ${ }^{1}$ Therefore these three solid forms of triol 2 could potentially be used as intermediates.

A different approach involved the isolation of a non-terminal alkyne that can be converted to parent triol 2 under mild reaction conditions. Crystalline TMS-triol $\mathbf{6}$ showed a melting point around $80{ }^{\circ} \mathrm{C}$, and exothermic decomposition starting around $226{ }^{\circ} \mathrm{C}$ with $1489 \mathrm{~J} / \mathrm{g}$ energy by DSC analysis. Although 6 is a highly energetic compound, the exotherm onset temperature is quite high, and drop weight testing indicated that it is not impact sensitive at an energy level of 29 J. Because the SADT (selfaccelerating decomposition temperature) for $50 \mathrm{~kg}$ was estimated to be greater than $75^{\circ} \mathrm{C}$, temperature control during shipping and storage is not required. Additionally, the purified solid is not hygroscopic as determined by DVS (dynamic vapor sorption) analysis. TMS-triol 6 was also characterized using single crystal x-ray diffraction (see SI for details). Preliminary TMS deprotection condition screenings revealed that TMS-triol 6 could be readily converted to triol 2 under aqueous conditions at elevated temperature or mild basic conditions. ${ }^{3}$ Further development of this reaction is described in the following paper of this issue. ${ }^{4}$ An additional advantage of this approach presented itself, as TMS-triol 6 could easily be extracted into organic solvents, providing a way to remove inorganic and highly aqueous soluble impurities by organic/aqueous extraction.

It should be noted that solid TMS-triol 6 has a minimum ignition energy of $<1 \mathrm{~mJ}$ with and without inductance. This means that the compound is extremely ignition sensitive and any electrostatic spark could ignite a dust cloud. Therefore, specific safety handling precautions have to be followed in order to handle the product safely.

In comparison with sodium salt $\mathbf{3}$ and DABCO co-crystal $\mathbf{4}$, the hemi-DABCO co-crystal $\mathbf{5}$ was slightly favored as it has a higher onset temperature for exothermic decomposition and is a more atom-economic alternative respectively. However, if synthetically accessible, TMS-triol 6 would be the preferred choice because of its improved physical properties including exotherm onset temperature and feasibility for organic/aqueous extraction.

Initial Route to Triol 2. As in previous syntheses of islatravir (1), ${ }^{5}$ the initial route to triol $\mathbf{2}$ which was used to prepare the material necessary for development of the biocatalytic cascade installed the alkyne through 1,2 addition into a protected form of a 1,3-dihydroxyketone. Using 1,3-dihydroxyacetone diacetate 7, prepared from acetyl protection of commerical dihydroxyacetone, as starting material allowed introduction of the ethynyl group using commercially available ethynylmagnesium chloride and isolation of alkyne $\mathbf{8}$ after aqueous workup as an oil in $74 \%$ yield (Scheme 2). Deprotection of the hydroxy groups could be achieved by reacting diacetate $\mathbf{8}$ in refluxing methanol in the presence of the acidic resin Amberlite IR-120 to yield triol 2. The material obtained after treatment of the crude reaction stream with activated carbon, filtration and removal of all volatiles had a purity of $56 \mathrm{wt} \%$. Triol 2 was further purified and isolated through crystallization as DABCO cocrystal $\mathbf{4}$, by treating a THF solution of crude triol 2 with DABCO.

Scheme 2. First Route for the Synthesis of Co-crystal 4 


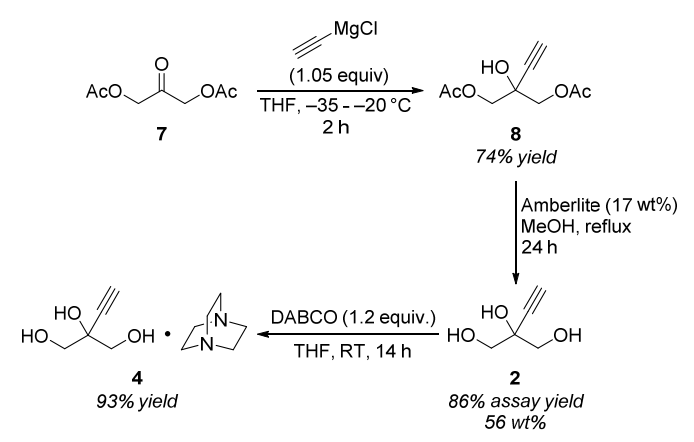

Efforts to streamline the synthesis focused on the use of tetrahydropyran (THP) as protecting group, as deprotection can be performed under milder reaction conditions (Scheme 3). Starting from inexpensive and readily-available dihydroxyacetone dimer 9, THP protection could be achieved in 93\% assay yield using 3,4-dihydropyran and catalytic pyridinium p-toluenesulfonate (PPTS) in toluene at $35^{\circ} \mathrm{C}$. The crude reaction mixture was added to a slight excess of ethynylmagnesium chloride in THF at a temperature slightly below $0{ }^{\circ} \mathrm{C}$ to afford alkyne $\mathbf{1 0}$. After an aqueous workup and concentration to remove THF, the crude solution was reacted with ethanol in the presence of the acidic resin Amberlyst 15 at $70{ }^{\circ} \mathrm{C}$ to remove the THP groups. The crude reaction stream was purified through a carbon treatment, partial solvent switch to THF and subsequent isolation of the DABCO co-crystal $\mathbf{4}$ as outlined above. This sequence afforded a yield of $47 \%$ from dihydroxyacetone dimer 9 without intermediate isolation and requiring one aqueous workup.

\section{Scheme 3. Through Process to DABCO Co-crystal 4}

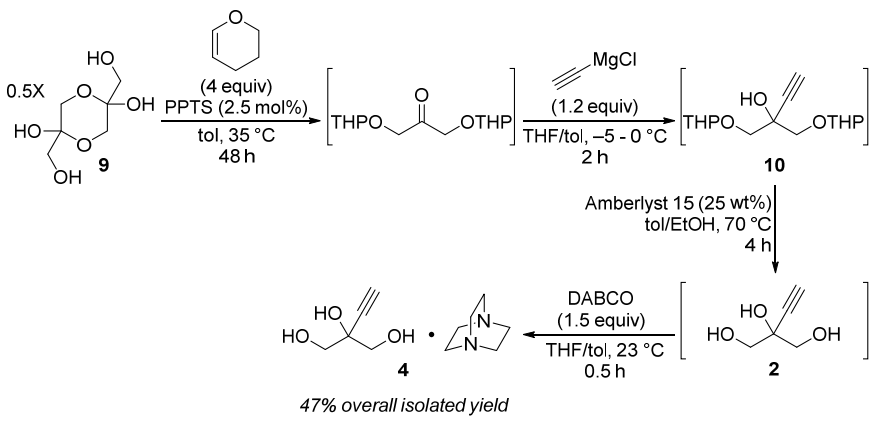

Route Scouting for the Manufacturing Route of Triol 2. While the route described above was sufficient to ensure material supply for the development of the biocatalytic cascade, several issues remained that should ideally be addressed in the manufacturing route: (1) upon quench of the acetylenic Grignard reagent used in the 1,2-addition, acetylene is generated. As acetylene is a highly explosive gas with a very low minimum ignition energy (MIE), a strict electrical code is required for its handling and venting in a manufacturing facility. Hence, acetylene use and generation presents a major safety concern, and ideally would be avoided. ${ }^{2}$ (2) Due to the introduction and removal of the protecting groups for the hydroxyl group, the synthetic sequence consists of three chemical steps while only one carbon-carbon bond is formed. A shorter route, ideally forgoing hydroxy protection, was therefore highly desirable. (3) The isolation of triol 2 through formation of a highly crystalline triolDABCO co-crystal $\mathbf{4}$ or $\mathbf{5}$ is a practical way to address the purity requirements and the lack of a crystalline intermediate throughout the route to triol 2 . Nevertheless, salt formation and salt break operations are necessary from the crude triol $\mathbf{2}$ stream.
Therefore, a new route should ideally allow a more straightforward way to an aqueous solution of triol $\mathbf{2}$, that meets the purity requirements of the subsequent biocatalytic cascade.

To address these challenges two main strategies were devised (Scheme 4). The first was based on the use of an alternative ketone starting material, which was monomeric and contained non-protic functional groups which could subsequently be converted to hydroxy groups, thereby allowing a direct 1,2-addition of an acetylide. To this end, oxetanone $\mathbf{1 1}$ and 1,3-dichloroacetone 12 were investigated. Starting from oxetanone 11, triol 2 was successfully generated through a two-step procedure (Scheme 4a). Following a literature procedure, ${ }^{6}$ 1,2-addition of ethynylmagnesium bromide into oxetane $\mathbf{1 1}$ afforded alkyne $\mathbf{1 3}$ as a crystalline solid in $98 \%$ yield. The oxetane was cleaved through subsequent treatment of alkyne $\mathbf{1 3}$ with the acidic resin Amberlite IR-120 in an aqueous solution, resulting in formation of the desired triol 2 in $82 \%$ yield. Despite the low step-count and high yield this route was deprioritized due to the high cost and low availability of the starting material 11 on commercial scale. Another route used 1,3-dichloroacetone $\mathbf{1 2}$ as starting material which was converted to alkyne 14 through 1,2-addition of a Grignard reagent obtained from TMS-acetylene (TMS = trimethylsilyl) and $\mathrm{MeMgBr}$. Subsequent treatment of the product 14 with aqueous $\mathrm{NaOH}$ yielded triol 2 in moderate yield, which could be improved to $>85 \%$ through phase transfer catalysis and the use of a milder carbonate base (Scheme 4b). Mechanistic studies of the reaction from dichloride 14 to triol 2 showed that the desilylation of the alkyne happened first, prohibiting isolation of TMS-triol 6. The alkyl chloride groups were cleaved under the same reaction conditions through generation of a series of epoxides and subsequent hydrolysis. From this reaction triol $\mathbf{2}$ could be isolated either as an aqueous solution or as hemi-DABCO co-crystal 4. This route offers a highyielding two-step access to the desired triol $\mathbf{2}$ while avoiding the use and generation of acetylene by employment of TMSacetylene as alkyne source. However, the high toxicity and lachrymatory nature of 1,3-dichloroacetone $\mathbf{1 2}$ prompted us to pursue another route.

\section{Scheme 4. Alternative Routes to Triol 2}

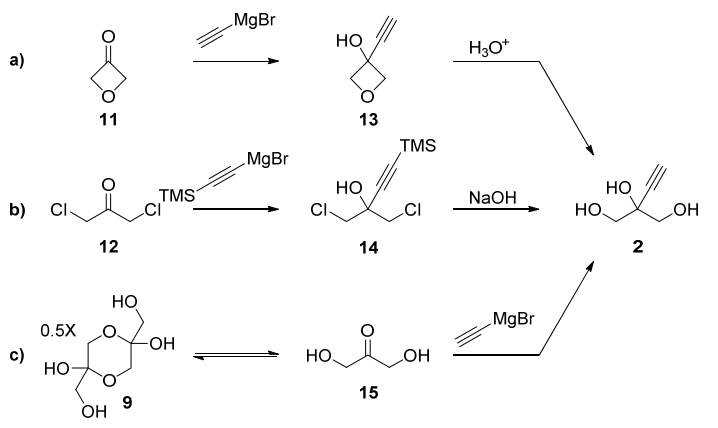

The second strategy targeted direct 1,2-addition of an alkyne into readily available 1,3-dihydroxyacetone dimer 9 (Scheme $4 c)$. Our investigations of this approach started with the screening a combination of bases and metal acetylides. For example, deprotonation of the hydroxyl groups with different bases including MeOK, EtOK, tBuOK, and LiHMDS in THF followed by addition of ethynylmagnesium bromide only yielded trace amounts of desired product $\mathbf{2}$. In addition, lithium, zinc, aluminum and manganese acetylides were prepared and studied in the reaction with dimer 9. However, under all the conditions 
screened, the desired triol product 2 was generated in less than $5 \%$ yield with the observation of significant decomposition of the starting material.

One of the main challenges for this approach was identified as the equilibrium between the dimer 9 and monomer 15 of 1,3dihydroxyacetone, as 1,2-addition was thought to only successfully occur on monomeric species 15. 1,3-Dihydroxyacetone can exist as a dimer or monomer in the solid state, although the latter slowly converts to the thermodynamically favored dimer. ${ }^{8}$ In our experience, commercial 1,3-dihydroxyacetone either consists of pure dimer or a monomer/dimer mixture even though it is sold as monomer. Dissolution of the dimer in protic solvents such as water and methanol shifts the solution equilibrium towards the monomer and allows for its isolation, although it reverts to the dimer upon storage. ${ }^{9}$ However, these solvents prohibit the use of most organometallic 1,2-addition protocols. In order to deconvolute issues with the monomer/dimer equilibrium from potential issues with a 1,2-addition on a substrate with two unprotected hydroxy groups, our early studies used isolated 1,3-dihydroxyacetone monomer $\mathbf{1 5}$ as starting material.

During high throughput experimentation (HTE) studies it was found that combinations of a dialkylzinc reagent with an amino alcohol or diamine ligand enabled addition of phenylacetylene into freshly prepared 1,3-dihydroxyacetone monomer 15. However, catalytic turnover could not be achieved and the reactions were stoichiometric in $\mathrm{Zn}$ (II) and ligand, rendering the approach unattractive. Further evaluation of the reaction was focused on the use of 1.5 equivalents of ethynylmagnesium bromide as the nucleophile and two equivalents of base to deprotonate the hydroxyl groups. The use of strong organic bases (e.g. LDA, LiHMDS, NaHMDS, KHMDS, n-BuLi) led to significant decomposition. Detectable amounts of desired product triol 2 were observed in reactions with alkoxide bases (KOMe and $\mathrm{NaOMe}$ ) or a sacrificial Grignard reagent (i$\mathrm{PrMgCl}$ ). However, significant decomposition of starting material was always observed under these conditions and the combined yield of starting material and desired product did not exceed $25 \%$. In contrast, the addition of 3 equiv. of ethynylmagnesium bromide to dihydroxyacetone monomer 15 generated $63 \%$ yield of desired triol product $\mathbf{2}$, likely because the magnesium acetylide had the appropriate balance of nucleophilicity and basicity to avoid decomposition experienced with other strong bases. Similar reactivity could also be observed with TMSethynylmagnesium bromide, offering a potential to avoid the generation of acetylene and isolation of the more preferred TMS-triol 6.

Based on these results the route involving direct 1,2-addition into dihydroxyacetone 9 (Scheme 4c) was pursued for further development.

Development of the Dihydroxyacetone Route. As mentioned above, proof of concept that direct 1,2-addition of an acetylenic Grignard into dihydroxyacetone monomer $\mathbf{1 5}$ is possible, was achieved through the use of 3 equiv. of ethynylmagnesium bromide as nucleophile and base to deprotonate the hydroxy groups, generating $63 \%$ yield of desired triol product 2 (Table 2, entry 1). Under the same conditions, reaction of dihydroxyacetone monomer 15 with TMS-ethynylmagnesium bromide proceeded smoothly and generated TMS-triol 6 in $80 \%$ yield (Table 2, Entry 2). When dihydroxyacetone dimer 9 was used as starting material addition product TMS-triol 6 was obtained in $45 \%$ yield (Table 2 entry 4 ), while a reaction with ethynylmagnesium bromide did not yield appreciable amounts of triol product 2 . (Table 2, entry 3 ) It should be noted that reactions with dimer 9 as starting material required longer reaction times when compared with reactions using monomer $\mathbf{1 5}$ as starting material.

Table 2. Acetylide Grignard addition to Dihydroxyacetone Dimer 9 and Monomer $15^{\text {a) }}$

\begin{tabular}{|c|c|c|c|c|}
\hline $\begin{array}{r}\mathrm{HO} \\
\times \quad 0\end{array}$ & 15 & $\frac{\mathrm{R}-\mathrm{MgBr}}{(3 \text { equiv.) }}$ & $\underbrace{\mathrm{HO} / / /}_{2} \mathrm{OH}$ & $\underbrace{\mathrm{HO} / /}_{6}$ \\
\hline Entry & Starting Material & Nucleophile & Reaction Time & Assay Yield \\
\hline 1 & & $\equiv \mathrm{MgBr}$ & $3 \mathrm{~h}$ & 2: $63 \%$ \\
\hline 2 & 15 & $\mathrm{TMS}=\mathrm{MgBr}$ & $3 \mathrm{~h}$ & 6: $80 \%$ \\
\hline 3 & & $\equiv-\mathrm{MgBr}$ & $18 \mathrm{~h}$ & 2: $0 \%$ \\
\hline 4 & ${ }_{\mathrm{OH}} \mathrm{O}$ & $\mathrm{TMS}=\mathrm{MgBr}$ & $18 \mathrm{~h}$ & $6: 45 \%$ \\
\hline
\end{tabular}

a) Reactions were carried out with addition of dihydroxy acetone dimer/monomer into Grignard reagents $(1 \mathrm{M}$ in THF, 3.0 equiv.) at $-20^{\circ} \mathrm{C}$ in 6 batches over $1 \mathrm{~h}$. The reactions were then warmed to $25^{\circ} \mathrm{C}$ and analyzed by quantitative-NMR.

Due to the direct accessibility from readily available dihydroxyacetone dimer 9, the feasibility to avoid generation of acetylene, the favorable physical properties, and the apparent ease of converting it to triol 2, TMS-triol 6 was chosen as an isolated intermediate en route to islatravir (1) and further development work focused on optimizing its synthesis. The key areas of improvement were identified to be efficiency, cycle time, and cost.

As TMS-ethynylmagnesium halide was the main contributor to the cost of the reaction, in-situ generation protocols were evaluated. One approach involved the use of 2 equiv. of lessexpensive Grignard reagents for the deprotonation of the hydroxy groups, thereby reducing the amount of more-expensive TMS-ethynylmagnesium halide needed. It was found that reactions with 2 equiv of $t$ - $\mathrm{BuMgCl}$ and only 1.5 equiv. of TMSethynylmagnesium bromide generated the desired product 6 in $44 \%$ yield, although at a much slower rate than with 3 equiv TMS-ethynylmagnesium bromide (120 h vs. $18 \mathrm{~h}$ ). No desired product 6 was observed when $i-\mathrm{PrMgCl}$ and $n$ - $\mathrm{HexMgCl}$ were used as bases. The TMS-ethynylmagnesium halide reagent could also be prepared directly from TMS-acetylene and $t$ $\mathrm{BuMgCl}$ giving TMS-triol $\mathbf{6}$ in moderate yields. Higher reaction temperatures $\left(45^{\circ} \mathrm{C}\right)$ helped to improve the reaction rate while an age-time of the in-situ TMS-ethynylmagnesium chloride generation greater than $10 \mathrm{~h}$ ensured consistent yields between $70-75 \%$. Using this optimized reaction protocol, a $40 \mathrm{~kg}$ scale reaction was successfully demonstrated and afforded $75 \%$ isolated yield, proving the robustness and scalability of the process. (Scheme 9)

\section{Scheme 9. Pilot plant demonstration}

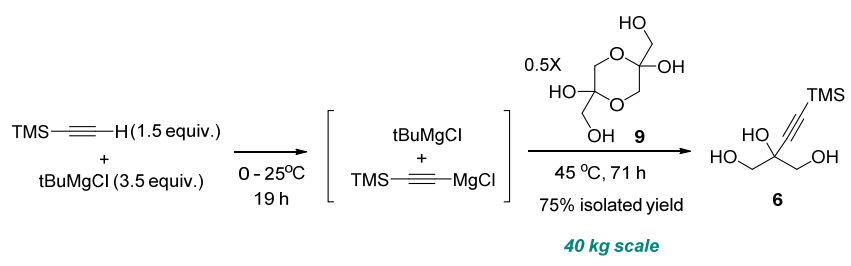


Alternatively, TMS-ethynylmagnesium chloride could be generated in-situ from $\mathrm{MeMgCl}$ and TMS-acetylene. Although in this way three equivalents of TMS-acetylene were necessary, the higher concentration and better commercial availability of $\mathrm{MeMgCl}$ outweighed the higher cost and was ultimately chosen for the manufacturing route. It was also observed that the resulting TMS-acetylenic Grignard reagent was stable for at least 7 days and performed without any diminished yield in the subsequent chemistry.

The slow equilibrium between dihydroxyacetone monomer 15 and dimer 9 was still thought to be the main reason for the long reaction times, as 1,2-addition likely only occurs on ketone 15. Our efforts therefore focused on optimizing the cracking of dimer 9 during or before the reaction and we turned to screening of acid-catalyzed conversion to monomer 15. A series of acids were examined where it was found that utilizing 1-2 mol\% TFA $(\mathrm{TFA}=$ trifluoroacetic acid) afforded the best results. Optimal conditions involved treating dimer 9 with 2 mol\% TFA in 10 $\mathrm{L} / \mathrm{kg}$ of THF at $60{ }^{\circ} \mathrm{C}$ for $1-2 \mathrm{~h}$. Initially, the reaction starts out as a slurry and becomes homogeneous once the dimer 9 was converted to monomer $\mathbf{1 5}$. The solution of monomer is stable above $40{ }^{\circ} \mathrm{C}$ for $24 \mathrm{~h}$ but will start to crystallize and convert back to dimer below $30{ }^{\circ} \mathrm{C}$. Solutions of monomer 15 obtained in this way could directly be used in the 1,2-addition, allowing much shorter reaction times.

The conditions chosen for manufacturing involved treatment of 3.2 equivalents of TMS-acetylene relative to the monomer 15 with 3.15 equivalents of $\mathrm{MeMgCl}$ at $30{ }^{\circ} \mathrm{C}$ and aging for 2 $\mathrm{h}$. The resulting solution was then added to a solution of monomer 15, prepared as described above from dimer 9 and catalytic amounts of TFA, followed by heating for $2-4 \mathrm{~h}$ at $55^{\circ} \mathrm{C}$. This resulted in full conversion to product 6 as determined by GC analysis of a quenched sample. The isolation of TMS-triol 6 involved inversely quenching into an aqueous solution of 3.4 equivalents of $3 \mathrm{M}$ aqueous $\mathrm{AcOH}$ and extraction with $10 \mathrm{~L} / \mathrm{kg}$ IPAc. After washing the organic layer with an $18 \mathrm{wt} \%$ solution of brine, the mixture was azeotropically dried and concentrated to a final volume of $7 \mathrm{~L} / \mathrm{kg}$ in IPAc which resulted in a slurry of the product. The internal temperature was adjusted to $45^{\circ} \mathrm{C}$ and heptane $(7 \mathrm{~L} / \mathrm{kg})$ was added over $4-5 \mathrm{~h}$. The resulting slurry was cooled to room temperature, followed by filtration which afforded a TMS-triol 6 in $80 \%$ isolated yield. Throughout the process acetylene was not detected in headspace of the reaction vessels by gas-phase Fourier-transform infrared (FT-IR) spectroscopy. This process has safely and successfully been conducted on $>100 \mathrm{~kg}$ scale at the commercial-scale manufacturing site. (Scheme 10).

Finally, TMS-triol 6 enters the biocatalytic cascade after removal of the TMS protecting group with catalytic Bis-Tris, providing triol 2 as an aqueous solution. Enzymatic phosphorylation then provides triol phosphate $\mathbf{1 6}$ in excellent conversion and ee. ${ }^{1}$

\section{Scheme 10. Final Process}

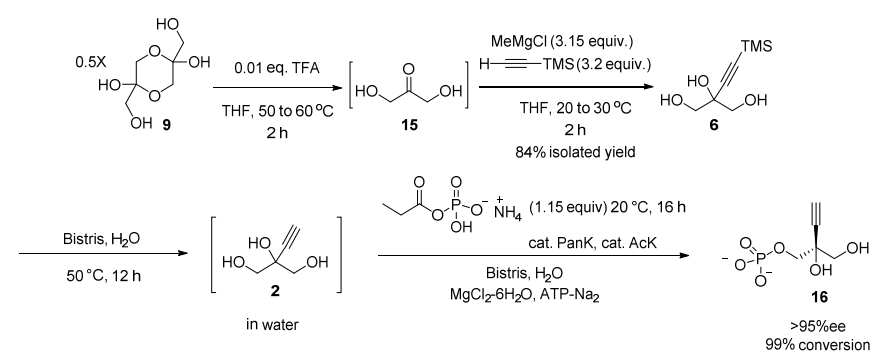

\section{Concluding Remarks}

This study reports the details behind the development of the synthesis to the 2-ethynyl glycerol building block $\mathbf{6}$. The route includes an in situ acid-catalyzed cracking of 1,3-dihydroxyacetone dimer and subsequent 1,2-addition of TMSethynylmagnesium chloride. The resulting product $\mathbf{6}$ is a crystalline and stable solid, addressing the concerns about unfavorable physical properties of triol $\mathbf{2}$ and derivatives. The route was succefully demonstrated on scale and directly used in the downstream biocatalytic cascade.

\section{EXPERIMENTAL SECTION}

General Considerations. All reactions were carried out under a nitrogen atmosphere. All solvents and reagents were used as received from commercial sources.

In a vessel was charged $146.6 \mathrm{~kg}$ of THF followed by $57.7 \mathrm{~kg}(587.6$ mol) of trimethylsilyl acetylene and the mixture was warmed to an internal temperature of $35^{\circ} \mathrm{C}$. To this solution was added $187.9 \mathrm{~kg}(559.8$ $\mathrm{mol}$ ) of $3 \mathrm{M} \mathrm{MeMgCl}$ at a rate of $0.63 \mathrm{~kg} / \mathrm{min}$ using a mass meter. The resulting mixture was stirred at $35^{\circ} \mathrm{C}$ for $4 \mathrm{~h}$ and was cooled to $20{ }^{\circ} \mathrm{C}$. In a separate vessel was added $134.7 \mathrm{~kg}$ of THF, $16.8 \mathrm{~kg}(93.3 \mathrm{~mol})$ of dihydroxyacetone dimer, and $213 \mathrm{~g}(1.87 \mathrm{~mol})$ of TFA. The slurry was heated to an internal temperature of $60^{\circ} \mathrm{C}$ for two hours and was cooled to $40{ }^{\circ} \mathrm{C}$ to give a homogeneous solution of dihydroxyacetone monomer. This solution was then added to the above Grignard solution at a maximum rate of $2.1 \mathrm{~kg} / \mathrm{min}$ using a mass meter. The vessel was follow-flushed with $15 \mathrm{~kg}$ of THF. The mixture was stirred at $20{ }^{\circ} \mathrm{C}$ for four hours and inversely quenched into $213.2 \mathrm{~kg}$ of a $3 \mathrm{M}$ aqueous solution of $\mathrm{AcOH}$. To the quenched mixture was added $146.5 \mathrm{~kg}$ of IPAc, the bi-phasic mixture mixed for $30 \mathrm{~min}$, and the layers were separated. The resulting organic solution was concentrated under reduced pressure of a final volume of $\sim 135 \mathrm{~L}$. To the solution was added $79.2 \mathrm{~kg}$ of IPAc and the distillation continued under reduced pressure to a volume of $\sim 135 \mathrm{~L}$. This flush was repeated 2 X 70 L IPAc and the distillation stopped at a final volume of $135 \mathrm{~L}$. The mixture was then warmed to $45^{\circ} \mathrm{C}$ and $80.4 \mathrm{~kg}$ of heptanes was charged over $1 \mathrm{~h}$. The mixture was then cooled to $20^{\circ} \mathrm{C}$, the resulting slurry was filtered and the wet cake washed with $115 \mathrm{~kg}$ of heptanes. The product was dried under vacuum/ $\mathrm{N}_{2}$ sweep for $38 \mathrm{~h}$ to provide $29.5 \mathrm{~kg}(84 \%)$ yield of TMS-triol 6 as a colorless solid. ${ }^{1} \mathrm{H}$ NMR $\left(400 \mathrm{MHz}, \mathrm{DMSO}-\mathrm{d}^{6}\right) \delta 5.12(\mathrm{~s}, 1 \mathrm{H})$, $4.65(\mathrm{t}, \mathrm{J}=6.1 \mathrm{~Hz}, 2 \mathrm{H}), 3.46-3.35(\mathrm{~m}, 4 \mathrm{H}), 0.12(\mathrm{~s}, 9 \mathrm{H}) .{ }^{13} \mathrm{C} \mathrm{NMR}(75$ MHz, DMSO-d $\left.{ }^{6}\right) \delta 0.2,64.9,71.5,87.2,109.1$. HRMS MS (m/z): [M $+\mathrm{Na}]+$ calcd for [C8H16O3Si Na] 211.0766; found 211.0766.

\section{AUTHOR INFORMATION}

\section{Corresponding Author* \\ *ji_qi@merck.com}

\section{ACKNOWLEDGMENT}

We thank Paul G. Bulger for his feedback on the manuscript and input on the project. Anna Fryszkowska is thanked for help with biocatalytic reactions as well as feedback on the manuscript and input on the project. We thank L.-C. Campeau, Benjamin D. 
Sherry, and Cheol K. Chung for their feedback on the manuscript. We thank François L. Levesque, Cecilia Bottecchia, and Timothy J. Wright for evaluating reactions in flow. We thank Mike Toth and Brittany Armstrong for experimental assistance with the safety evaluation. We thank Zhanqiang Guo, Yongwei Wu, Shunjia Zheng, Guiquan Liu, Jun Zhang, Jianfeng Zhan, Wanyong Fu, Minyi Feng, and Fang Wang for experimental help.

\section{REFERENCES}

(1) Huffman, M. A.; Fryszkowska, A.; Alvizo, O.; Borra-Garske, M.; Campos, K. R.; Canada, K. A.; Devine, P. N.; Duan, D.; Forstater, J. H.; Grosser, S. T.; Halsey, H. M.; Hughes, G. J.; Jo, J.; Joyce, L. A.; Kolev, J. N.; Liang, J.; Maloney, K. M.; Mann, B. F.; Marshall, N. M.; McLaughlin, M.; Moore, J. C.; Murphy, G. S.; Nawrat, C. C.; Nazor, J.; Novick, S.; Patel, N. R.; Rodriguez-Granillo, A.; Robaire, S. A.; Sherer, E. C.; Truppo, M. D.; Whittaker, A. M.; Verma, D.; Xiao, L.; $\mathrm{Xu}, \mathrm{Y}$.; Yang, H. Design of an in Vitro Biocatalytic Cascade for the Manufacture of Islatravir. Science 2019, 366 (6470), 1255-1259. https://doi.org/10.1126/science.aay8484.

(2) Schobert, H. Production of Acetylene and Acetylene-Based Chemicals from Coal. Chem. Rev. 2014, 114 (3), 1743-1760. https://doi.org/10.1021/cr400276u.

(3) Full conversion of TMS deprotection of $\mathbf{1 9}$ was observed in water at $70{ }^{\circ} \mathrm{C}$ for $3 \mathrm{~h}$ or with $10 \mathrm{wt} \%$ Ambersep ${ }^{\circledR} 900$ at ambient temperature for $3 \mathrm{~h}$.

(4) Further details on the phosphorylation will be described in a future publication.

(5) (a) Nawrat, C. C.; Whittaker, A. M.; Huffman, M. A.; McLaughlin, M.; Cohen, R. D.; Andreani, T.; Ding, B.; Li, H.; Weisel, M.; Tschaen, D. M. Nine-Step Stereoselective Synthesis of Islatravir from Deoxyribose. Org. Lett. 2020, 22 (6), 2167-2172. https://doi.org/10.1021/acs.orglett.0c00239. (b) Patel, N. R.; Nawrat, C. C.; McLaughlin, M.; Xu, Y.; Huffman, M. A.; Yang, H.; Li, H.; Whittaker, A. M.; Andreani, T.; Lévesque, F.; Fryszkowska, A.; Brunskill, A.; Tschaen, D. M.; Maloney, K. M. Synthesis of Islatravir Enabled by a Catalytic, Enantioselective Alkynylation of a Ketone.
Org. Lett. 2020, 22 (12), 4659-4664. https://doi.org/10.1021/acs.orglett.0c01431. (c) Patel, N. R.; Huffman, M. A.; Wang, X.; Ding, B.; McLaughlin, M.; Newman, J. A.; Andreani, T.; Maloney, K. M.; Johnson, H. C.; Whittaker, A. M. Five-Step Enantioselective Synthesis of Islatravir via Asymmetric Ketone Alkynylation and an Ozonolysis Cascade. Chem. $\quad-\quad$ Eur. J. n/a (n/a). https://doi.org/10.1002/chem.202003091. (d) McLaughlin, M.; Kong, J.; Belyk, K. M.; Chen, B.; Gibson, A. W.; Keen, S. P.; Lieberman, D. R.; Milczek, E. M.; Moore, J. C.; Murray, D.; Peng, F.; Qi, J.; Reamer, R. A.; Song, Z. J.; Tan, L.; Wang, L.; Williams, M. J. Enantioselective Synthesis of 4'-Ethynyl-2-Fluoro-2'-Deoxyadenosine (EFdA) via Enzymatic Desymmetrization. Org. Lett. 2017, 19 (4), 926-929. https://doi.org/10.1021/acs.orglett.7b00091. (e) Kageyama, M.; Miyagi, T.; Yoshida, M.; Nagasawa, T.; Ohrui, H.; Kuwahara, S. Concise Synthesis of the Anti-HIV Nucleoside EFdA. Biosci. Biotechnol. Biochem. 2012, 76 (6), $1219-1225$. https://doi.org/10.1271/bbb.120134. (f) Kageyama, M.; Nagasawa, T.; Yoshida, M.; Ohrui, H.; Kuwahara, S. Enantioselective Total Synthesis of the Potent Anti-HIV Nucleoside EFdA. Org. Lett. 2011, 13 (19), 5264-5266. https://doi.org/10.1021/ol202116k.

(6) Wang, Z.; Chen, Z.; Sun, J. Catalytic Enantioselective Intermolecular Desymmetrization of 3-Substituted Oxetanes. Angew. Chem. Int. $\quad E d . \quad 2013, \quad 52 \quad$ (26), 6685-6688. https://doi.org/10.1002/anie.201300188.

(7) Chênevert, R.; Simard, M.; Bergeron, J.; Dasser, M. Chemoenzymatic Formal Synthesis of (S)-(-)-Phosphonotrixin. Tetrahedron Asymmetry 2004, $15 \quad$ (12), 1889-1892. https://doi.org/10.1016/j.tetasy.2004.04.046.

(8) Davis, L. The Structure of Dihydroxyacetone in Solution. Bioorganic Chem. 1973, 2 (3), 197-201. https://doi.org/10.1016/00452068(73)90023-0.

(9) Insight into the Unexpectedly Rapid Degradation of Dihydroxyacetone-Based Hydrogels - Ricapito - 2016 - Macromolecular Chemistry and Physics - Wiley Online Library https://onlinelibrary.wiley.com/doi/10.1002/macp.201600170 (accessed Oct 8, 2020). 